\title{
The perfect personalized cancer therapy: cancer vaccines against neoantigens
}

\author{
Luigi Aurisicchio ${ }^{1,2^{*}}$ (D) Matteo Pallocca ${ }^{3}$, Gennaro Ciliberto ${ }^{4}$ and Fabio Palombo ${ }^{1,5}$
}

\begin{abstract}
In the advent of Immune Checkpoint inhibitors (ICI) and of CAR-T adoptive T-cells, the new frontier in Oncology is Cancer Immunotherapy because of its ability to provide long term clinical benefit in metastatic disease in several solid and liquid tumor types. It is now clear that $\mathrm{ICl}$ acts by unmasking preexisting immune responses as well as by inducing de novo responses against tumor neoantigens. Thanks to theprogress made in genomics technologies and the evolution of bioinformatics, neoantigens represent ideal targets, due to their specific expression in cancer tissue and the potential lack of side effects. In this review, we discuss the promise of preclinical and clinical results with mutation-derived neoantigen cancer vaccines (NCVs) along with the current limitations from bioinformatics prediction to manufacturing an effective new therapeutic approach.
\end{abstract}

\section{Background}

Even though cancer therapy has made significant advances in the last decade, in the majority of cases it still fails to achieve long-lasting responses in patients with metastatic disease. To explain the reasons why tumors relapse the clonal evolution model has been proposed to reveal how intra-tumor heterogeneity $(\mathrm{TH})$ is the basis for emerging tumor variants under targeted therapies and immunological pressures [1].

The use of next generation sequencing (NGS) for massive analysis of cancer genomes allows a quantitative measurement of mutational frequencies and genome copy variations. The cancer atlas is quite diverse, ranging from a few to thousands of mutations for individual histological tumors [2], thus raising concerns on how to deal with this high complexity. Mutations are classified according to their role in tumor growth. Most of them do not confer intrinsic growth advantage and are defined "passenger mutations" whereas a smaller number of them, known as "driver mutations", provide a growth advantage and are therefore selected during tumor evolution. Druggable mutations, a subset of driver mutations, are defined by the availability of a drug (or the possibility to generate a drug)

\footnotetext{
* Correspondence: aurisicchio@takisbiotech.it

Luigi Aurisicchio and Matteo Pallocca contributed equally.

Gennaro Ciliberto and Fabio Palombo contributed equally.

${ }^{1}$ Takis, Rome, Italy

${ }^{2}$ Biogem, Ariano Irpino, Italy

Full list of author information is available at the end of the article
}

capable of targeting a specific genomic alteration. An intense research activity has currently been launched towards extending the use of such drugs to most tumor types which carry a selected mutation. Most of these mutations encode amino acid substitutions and therefore are collectively known as nonsynonymous mutations, resulting in new, cancer-specific protein sequence not expressed in normal tissues.

The analysis of different regions of the same tumor revealed that some mutations are commonly present (clonal) while others are unique only in some parts of it (subclonal) contributing to TH. High $\mathrm{TH}$ may explain why initial clinical responses defined by the reduction of tumor mass can fail at later times due to the outgrowth by treatment-resistant cancer subpopulations. It is important to stress that under selective pressures, tumor evolution can be redirected according to the timing and type of cancer therapy [3]. Ideally, we need to combine therapies against as many possible tumor-specific targets in order to reduce the likelihood of emerging escape variants. Small molecule inhibitors as well as biologics raised against driver/actionable mutations are designed against one target at the time, requiring a long development process, which results in a limited available armamentarium with a series of related side-effects. In this scenario, the feasibility of a multivalent target therapy made of small molecules or biologics is limited by practical reasons and cumulative side-effects associated with therapeutic drugs. 
The promise of a personalized cancer vaccine is therefore to target multiple tumor specific mutations reducing side-effects by sparing normal tissue and keeping tumors under immunological memory control for as long as possible. In this review, we describe the mechanisms underlying the basis of immune recognition of tumor cells and the evidence of preclinical and clinical studies in the emerging field of mutation-derived neoantigen cancer vaccines.

\section{T-cell immune response against self- and non-self antigens}

T-cells are capable to recognize and kill cells presenting on their surface non-self or altered self-antigens, i.e. peptides derived from intracellular protein cleavage. Proteins are cleaved by the proteasome generating a peptide pool, which is loaded into the endoplasmic reticulum by the TAP-1 system. In order to be presented on the cell surface, peptides are further trimmed and complexed with major histocompatibility complex (MHC; also known as human leukocyte antigen - HLA - in humans) class I molecules for their presentation to CD8+ T cells. MHC-I is a heterodimer composed of a polymorphic heavy chain and $\beta 2$-microglobulin. Peptides are also presented by MHC-class II molecules when they are digested through autophagy. MHC-II complexes are exposed to the immune system by antigen presenting cells (APC), such as dendritic cells (DC), and upon IFN- $\gamma$ stimulation also by other cell types including epithelial cells [4]. MHC-II presented peptides derived from proteins digested in the endocytic pathway are recognized by CD4+ T-cells. The subset of peptides capable of stimulating $\mathrm{T}$-cells are defined antigens.

Decades of research have led to the identification of a large number of self tumor antigens derived from the processing of normal proteins that have been grouped into three categories: tumor associated antigens (TAAs), tumor specific antigens (TSAs) and cancer testis antigens (CTAs). TAAs are defined as those antigens overexpressed by cancer cells than normal tissues. TSAs are those specifically expressed only in cancer cells and not in normal tissues. CTAs are expressed, besides tumor cells, only in germline tissues and trophoblastic cells [5]. These antigens have been the focus of intense preclinical and clinical research in the attempt to generate therapeutic cancer vaccines targeting these antigens. Unfortunately, in spite of encouraging pre-clinical data, a lifetime worth of clinical cancer research with these antigens has led to the conclusion that breaking immunological tolerance against self-antigens is actually more difficult than originally anticipated. In the meantime, the massive use of "omics" in cancer research has revealed that non-self-antigens derived from non-synonymous mutations in the coding region of proteins are instead efficiently recognized by the T-cell specific immune response (reviewed in $[5,6]$ ). In this review, we will not discuss antigens derived from post translational modifications as it has recently been published in a paper [7] but only mutation-derived ones that we will refer to as neoantigens.

Several lines of evidence support neoantigens as being important targets for immune responses. A higher neoantigen load was indeed associated with improved patient survival in a study that assessed hundreds of tumors with 6 different histological types from the TCGA [8]. An association between neoantigen load, increased number of tumor infiltrating lymphocytes (TILs) and improved survival was observed in colorectal [9] and endometrial cancer [10]. Neoantigen-specific T-cell immunity correlates with clinical response to immune checkpoint inhibitors (ICI) [11].

Monoclonal antibodies interfering with the programmed cell death protein 1 (PD1) and cytotoxic $\mathrm{T}$ lymphocyte antigen 4 (CTLA-4) signaling pathway are effective in many solid and hematological malignancies leading the FDA to approve their use in a growing list of tumors with different types of histology [12]. The clinical response to ICI treatment indeed correlates with neoantigen load in patients with melanoma [13], non-smallcell lung cancer (NSCLC) [14], and colorectal cancer [15]. Moreover, neoantigen-specific T-cell responses become evident in patients treated with ipilimumab (anti-CTLA-4) and with pembrolizumab (anti PD1). Although high neoantigen load is associated with good prognosis, the nature of tumor mutations is also relevant for the therapy based on ICI [16]. High levels of TH is associated with resistance and tumor escape [16]. A possible explanation of this may be the limited number of responses against neoantigens observed in patients treated with ICI as compared to the neoantigen repertoire presented by tumor cells [17]. Finally, in a separate set of observations with adoptive T-cell transfer, patients with solid tumors showed measurable T-cell specific immune responses against neoantigens $[18,19]$. On this basis, neoantigen cancer vaccines (NCVs) may represent an emerging new clinical approach to treat cancer.

\section{NCVs in preclinical tumor models}

NCVs have proven to be effective in different preclinical animal models (Table 1). The current method used to identify neoantigens and generate NCVs [20] is based on the following three steps (Fig. 1): 1) Collection of tumor and normal samples; 2) identification of neoantigens; 3) formulation of the vaccine. In the mouse system, nonsynonymous tumor-specific point mutations are identified by comparison of exome sequencing data of the tumor cell line of interest with reference to the mouse genome. In order to be immunogenic, a neoantigen has 
Table 1 Preclinical data with NCV

\begin{tabular}{lllll}
\hline $\begin{array}{l}\text { Type of neoantigen } \\
\text { vaccine }\end{array}$ & Formulation & Type of Tumor & $\begin{array}{l}\text { Checkpoint Inhibitor } \\
\text { Blocked }\end{array}$ & Anti tumor effects \\
\hline $\begin{array}{llll}\text { peptides } \\
\text { peptides }\end{array}$ & poly(l:C) & - & tumor delay \\
& $\begin{array}{l}\text { anti-CD40 antibody }+ \\
\text { poly(l:C) }\end{array}$ & Colanoma & - & tumor delay \\
peptides & - & Fibrosarcoma & - & tumor delay \\
peptides & poly(l:C) & Sarcoma & anti PD-1 & tumor delay \\
peptides & poly(l:C) & sarcoma & - & tumor delay \\
RNA and peptides & RNA complexed with & Colon, melanoma, mammary & - & tumor delay \\
& cationic lipids & carcinoma & & [25] \\
RNA & RNA complexed with & Colon & - & tumor delay \\
cationic lipids & [63] \\
peptides & poly(l:C) & ovary & - & No efficacy \\
peptides & lipoprotein-mimicking & colon, melanoma & anti PD-1 & tumor delay and tumor \\
& nanodiscs & & eradication \\
Long peptides & poly(l:C) & Head and neck cancer & - & tumor delay \\
\hline
\end{tabular}

to be expressed. Therefore mutations are further selected according to the level of gene expression measured by RNA-seq. Finally, the expressed neoantigens are ranked according to different bioinformatic pipelines as described below. The most popular methods to predict binding to MHC are NetMHC-4 and NetMHCpan
[21]. The last step is the delivery of neoantigens in an immunogenic formulation that includes peptides complexed with adjuvants [20] or with liposome particles [22] or delivered as an RNA vaccine [22]. This workflow results in cancer-specific immune responses that are efficacious against several tumor types including melanoma,

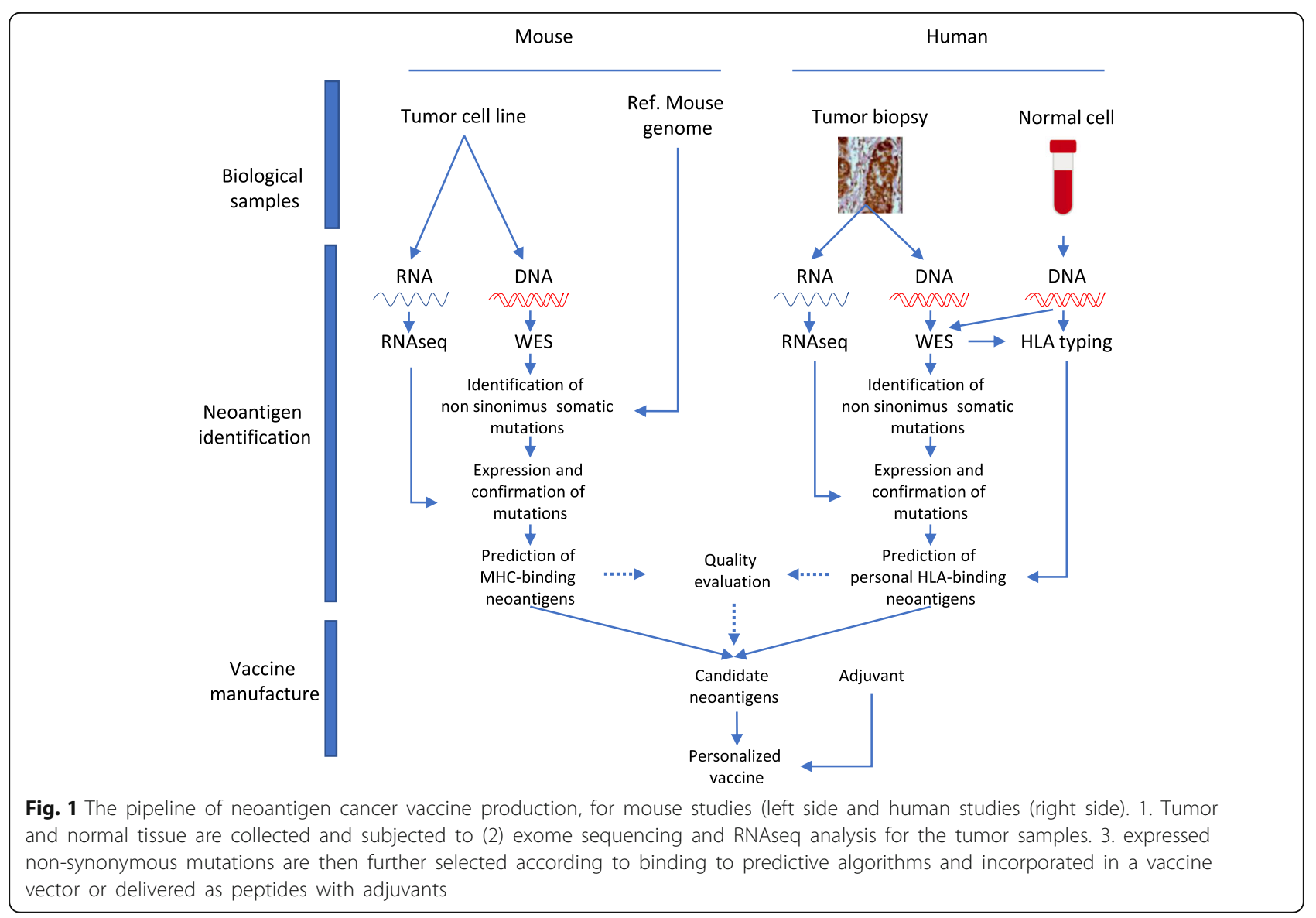


colon cancer and sarcoma (Table 1). The pipeline for $\mathrm{NCV}$ production in preclinical mouse models can be further refined by the introduction of immunoproteomic methods designed to discover neoantigens associated with MHC-I complex as it was shown in a colon cancer model [23]. The validity of the neoantigens identified by this approach was further supported by the confirmation of the immune responses in a subsequent work where the neoantigens were successfully utilized with a different vaccination platform [24].

NCVs-induced immune responses are in most cases specific for the neoantigens. The initial study provided evidence of responses with some cross-reactivity to wild type cognate epitopes measured by ELIspot assay [20]. On the contrary, subsequent papers showed a more stringent specificity for neoantigens likely due to the use of shorter peptides for flow cytometry analysis and the employment of dextramer staining for the detection of neoantigenspecific T-cells [23-27]. The most surprising evidence emerging from mouse studies is the observation that NCV induces not only a CD8+ but also a CD4 + T-cell response, and that the $\mathrm{CD} 4^{+} \mathrm{T}$-cell response is primarily responsible for the therapeutic effects [22]. This observation has initially been described using an innovative RNA vaccination platform [28] and was later confirmed by an independent group, which utilized a vaccine based on peptides [29]. Only one study combined NCV with anti-PD1 treatment [29]. This study suggests an additive effect of $\mathrm{NCVs}$ and immunotherapy on tumor growth inhibition. Notably, one report did not show antitumor activity in an ovarian cancer model despite the induction of a significant T-cell specific response against neoantigens [30]. The authors highlighted the limited number of mutations in this tumor type and the lack of high-affinity neoantigens, which may be detrimental for an effective NCV approach. A recent paper explored a head and neck cancer model providing further evidence that $\mathrm{NCV}$ is able to prevent tumor growth [31].

Whilst these initial studies describing different vaccination platforms and detection systems consistently support NCVs as a promising approach, some questions still remain unanswered. The first is that it is not clear whether the same neoantigen sequences are equally effective using different vaccination methods. It is worth mentioning that prediction of immunogenicity is mostly based on peptide vaccines that may be not informative for other vaccination platforms. Immunodominant epitopes may rank differently or even may not be confirmed in a context dependent manner. Our experience and observations from other groups in the field suggests that further research is needed to determine how vaccination technologies impact the quality of the immune response. It would be useful to generate a comprehensive neoantigen database that takes into account all the steps for the
NCVs process including the delivery method and the resulting immune responses in order to improve prediction models. A second question concerns the potential cross-reactivity of neoantigens with wild-type sequences. In this case immunological potency may be limited by central and peripheral tolerance leading to an ineffective T-cell response against the tumor. This class of neoantigens may, therefore, be more similar to the classical TAAs and may result in lower immunogenicity. In addition, vaccination with this group of neoantigens may cause potential side-effects against normal tissues, particularly when a vaccine could contain several crossreacting neoantigens, which can lead to cumulative sideeffects. To be on the safe side we suggest to exclude them from the design of a NCV.

\section{NCVs in clinical trials}

The efficacy of targeting tumor-specific non-self-antigens has been demonstrated in the case of cervical cancer driven by HPV [32, 33]. The immunogenicity of HPV is well documented by prophylactic HPV vaccines, proven to be effective in preventing cervical cancer in young adolescents. For the therapeutic approach the vaccine has to target a different group of viral proteins, namely the oncogenic E6 and E7. A plasmid DNA encoding HPV oncogenic proteins was administered in conjunction with electroporation as the delivery method to induce CD8+ effector T-cells. Targeting key viral proteins E6/E7 resulted in reduction or stabilization of cervical intraepithelial neoplasia (CIN) $2 / 3$ in $50 \%$ of patients [33] and in specific immune responses against the HPV targets [34]. In contrast, a similar vaccine technology delivering a fusion protein made of a self-TAA fused to an immunogenic bacterial antigen resulted in immune responses measured only against the non-self portion of the antigen, further supporting the idea that non-self antigens are immunogenic even in potentially immunocompromised patients with high tumor burden [35].

The design of mutation-derived tumor-specific NCVs in human clinical trials recapitulates the mouse protocol with some additional steps (Fig. 1). Tumor biopsy analyses are in fact much more complex than cancer cell lines (as reported in mouse studies) and in most cases the use of formalin fixed paraffin embedded slices as a source material is a factor for a good quality RNA-seq. For some tumors, the low amount of tumor material requires an increased sequencing depth to reveal the presence of rare cancer mutations. Reference normal tissue, usually available as blood samples, serves not only to compare tumor genome with the aim to identify somatic mutations but also to establish the individual HLA. The highly polymorphic nature of the HLA locus poses an issue for the prediction of neoantigens, since limited information is available for rare HLA. Although the prediction pipeline 
requires additional bioinformatic work, many tools are already available on the web and moreover clinical trials with cancer-specific neoantigens have been reported in melanoma patients using different vaccination strategies [36-38]. Three HLA-A2.1 positive melanoma patients, who had been pretreated with ipilimumab, were vaccinated with DC loaded with peptides encompassing the neoantigen mutations (NCT00683670) [36]. Predicted neoantigens were further selected according to a binding assay using HLA-A2.1 expressing T2 cells and seven validated peptides were used for each patient. Immune responses were detected in all patients although the assay required an in vitro growth of T cells with IL-2. The vaccine expanded T-cells against preexisting dominant epitopes and induced new responses, which were absent before treatment. More recently, a second clinical trial with peptide vaccines has been reported (NCT01970358) [37]. Six naive melanoma patients were vaccinated with a pool of synthetic long peptides + adjuvant. Up to 20 neoantigens were injected in 4 different sites upon formulation with poly-dIdC. Overall, the authors confirmed specific T-cell responses for 24 out of 28 neoantigens. Most of the responses were mediated by $\mathrm{CD} 4^{+} \mathrm{T}$-cells, however none of the neoantigen-specific $\mathrm{T}$-cells recognized cultured tumor cells in four out of six patients. The two patients with stage IV M1b relapsed after the last vaccination and were treated with anti-PD1. Both showed a clinical response, although the response rate in this subgroup of patients treated with ICI is expected to be only 61\%. Upon ICI treatment, new CD4 and CD8 responses against neoantigens were observed. In a third study (NCT02035956) [38], the vaccination with RNA was effective in inducing strong neoantigen-specific CD4 and CD8 responses in 13 melanoma patients in line with previous mouse evidence from the same research group [22]. Eight patients remained tumor free for the follow up period (12/24 months) whereas five patients relapsed during immune therapy. One patient was treated with a combination of NCV and ICI, with a good response. A second patient did not respond to NCVs/ICI and died. In this patient, the analysis of recurring metastasis showed the biallelic loss of $\beta 2$ microglobulin as the explanation for the lack of tumor response. The predominant CD4 response was also evident for the RNA based vaccination in addition to a relevant percentage of promiscuous double positive $\mathrm{CD} 4$ and $\mathrm{CD} 8$ neoantigens.

These results taken together suggest that NCVs may turn out to be a suitable clinical approach for highly heterogeneous tumors providing the best balance/ratio between targeting tumors (specificity) while sparing normal tissue (toxicity). However, confirmatory data in larger studies are needed to confirm. Indeed, several active clinical trials with NCVs are ongoing (see https://clinicaltrials.gov/) with different vaccination technologies and targeting different cancers. The most common are basket trial targeting different tumor histology (NCT02992977, NCT03289962, NCT 02897765) or lung cancer (NCT02956551, NCT03380871, NCT03166254), followed by glioblastoma (NCT03422094, NCT02287428) and disease-specific trials (Table 2).

\section{Bioinformatic methods for neoantigen prediction}

One of the main issues for NCVs development is the correct prediction of neoantigens. Several bioinformatic tools have been designed in order to call putative neoantigens from genomic data (https://www.ncbi.nlm.nih. gov/pubmed/27376489). The increasing interest in this matter is proven by the fact that 5 out of 7 publicly available pipelines were presented last year.

Neoantigen prediction involves a series of computational steps that can be inferred with specific experimental techniques (Fig. 1). It is for this reason, that bioinformaticians in previous years have focused on creating specialized software for specific sub-tasks (e.g. HLA typing from sequences as well as allele specific expression tools, [39-42], or fit-forall environments with complex pipelines that address several, or even all, analytical tasks (Table 3). Table 3 contains packages that are meant to be "plug and play" even if the installation process of such a framework can be cumbersome. Furthermore, to our knowledge, there is no freely and publicly available cloud web tool able to process all the required steps for neoantigen prediction from genomic data (https://www.ncbi.nlm.nih.gov/pubmed/27376489).

The typical steps of a neoantigen extraction method starts with the computation of allele-specific coverage. The algorithms typically use aligned sequence data from total RNA-seq and a list of variants from exome/genome sequencing to infer the relative wild type/mutant expression levels at base/mutation level of resolution. With this output it is possible to compute the mutated protein sequence through dedicated software for the assignment of the mutation to the correct protein. The predicted epitopes are then processed with prediction methods that rank the epitopes for binding affinity. This simple three step process (allele coverage/sequence translation/ binding prediction) contains several caveats that can hinder the whole process by calling false positives (nonexistent epitopes) or false negatives (missed epitopes).

In the translation process, it is obviously of utter importance to choose the right transcript isoform to translate. This step is not so obvious when the mutant allele coverage is computed at the base level, i.e. it is required to understand which of the overlapping expressed isoforms harbor that mutation. If the computation of the exact transcript results to be a process too cumbersome, a decent tradeoff is to choose the dominant transcript for the putative neoantigen identification since it has been shown that most highly expressed genes have one dominant isoform [43]. 
Table 2 NCV studies in clinical development trial

\begin{tabular}{lllll}
\hline Clinical trial & Cancer & Intervention & vaccine & status \\
\hline NCT01885702 & Colorectal Cancer & DC vaccination & DC & Active, not recruiting \\
NCT02632019 & Biliary tract tumor & DC vaccination and gemcitabine & DC & Unknown status \\
NCT02956551 & Lung cancer & DC vaccination & DC & Not yet recruiting \\
NCT03122106 & Pancreatic Cancer & Neoantigen DNA vaccine with electroporation & DNA & Recruiting \\
NCT03199040 & Triple Negative Breast Cancer & Neoantigen DNA vaccine and Durvalumab & DNA & Not yet recruiting \\
NCT01970358 & Melanoma & Peptides with Poly-ICLC & peptide & Active, not recruiting \\
NCT03068832 & Pediatric Brain Tumor & Peptide vaccine with Poly ICLC & peptide & Not yet recruiting \\
NCT02287428 & Glioblastoma & NeoAntigen Vaccine and Radiation Therapy & peptides & Active, not recruiting \\
NCT02897765 & Many & NEO-PV-01 and Nivolumab & peptides & Recruiting \\
NCT02950766 & Kidney Cancer & Drug: NeoVax|Drug: Ipilimumab & peptides & Not yet recruiting \\
NCT03166254 & Lung Cancer & Long peptide with Poly ICLC and Pembrolizumab & Peptides & Not yet recruiting \\
NCT03219450 & Lymphocytic Leukemia & NeoVax with Cyclophosphamide & peptides & Not yet recruiting \\
NCT03359239 & Urothelial Bladder Cancer & Peptides with Poly ICLC and Atezolizumab & peptides & Not yet recruiting \\
NCT03361852 & Follicular Lymphoma & Neo Vax and Rituximab & peptides & Not yet recruiting \\
NCT03380871 & Lung Cancer & NEO-PV-01 Pembrolizumab Carboplatin Pemetrexed & peptides & Not yet recruiting \\
NCT03422094 & Glioblastoma & NeoVax and Nivolumab Ipilimumab & Peptides & Not yet recruiting \\
NCT03289962 & Many & Drug: RO7198457|Drug: Atezolizumab & RNA & Recruiting \\
NCT02992977 & many & AutoSynVax TM vaccine & HSP Peptides Active, not recruiting \\
\hline
\end{tabular}

Another issue related to transcript identification is the relative abundance of expression, inferable from the normalized coverage, since a reasonable choice would be not to include epitopes that are poorly expressed. The threshold for "low abundance expression" is a matter of discussion in the bioinformatic community involved in RNA-seq data analysis. Since an expression level of FPKM (Fragments Per Kilobase of transcript per Million mapped reads) between 1 and 5 represents around 1 transcript copy per cell, the most reasonable way of proceeding would be to eliminate all epitopes generating from isoforms of FPKM < 5. At the base level, since there is no accepted threshold for the RPM expression level of the mutation itself. Hence, one possibility may be to adhere to the transcript FPKM filter and to a high relative MUT/WT ratio.

The authors themselves have implemented a simple method called NaRciSo, in order to extract a list of expressed epitopes from paired Exome and RNA-seq data or standalone RNA-seq (manuscript in preparation). One of its modules is meant to predict neoantigens in the absence of exome sequencing data, computing a "RNA VCF" from RNA-seq sequence data and fetching it to the allele counter package.

Finally, to our knowledge the current available prediction tools that process from sequence reads to

Table 3 Pipelines for neoantigen prediction

\begin{tabular}{|c|c|c|c|c|}
\hline Title & Input & Notes & Date & Ref \\
\hline $\begin{array}{l}\text { TIminer: NGS data mining pipeline for cancer } \\
\text { mmunology and immunotherapy. }\end{array}$ & RNA-seq BAM and VCF & Computes GSEA and IPS & $10 / 2017$ & {$[64]$} \\
\hline $\begin{array}{l}\text { CloudNeo: a cloud pipeline for identifying } \\
\text { patient-specific tumor neoantigens. }\end{array}$ & BAM for HLA and VCF & $\begin{array}{l}\text { Computes HLA type and } \\
\text { Neoantigens }\end{array}$ & $10 / 2017$ & [65] \\
\hline $\begin{array}{l}\text { TSNAD: an integrated software for cancer } \\
\text { somatic mutation and tumour-specific } \\
\text { neoantigen detection. }\end{array}$ & FASTQ; BAM for HLA & Neoantigen detection pipeline & 05/2017 & [66] \\
\hline $\begin{array}{l}\text { INTEGRATE-neo: a pipeline for personalized } \\
\text { gene fusion neoantigen discovery. }\end{array}$ & FASTQ & $\begin{array}{l}\text { Gene fusion prediction and } \\
\text { neoantigen computation from } \\
\text { gene fusions }\end{array}$ & $02 / 2017$ & [67] \\
\hline $\begin{array}{l}\text { pVAC-Seq: A genome-guided in silico } \\
\text { approach to identifying tumor neoantigens. }\end{array}$ & $\begin{array}{l}\text { prepare FASTA (prepare input) } \\
\text { and predicts neoantigens }\end{array}$ & $\begin{array}{l}\text { Neoantigen calling, HLA typing, } \\
\text { MHC binding }\end{array}$ & $01 / 2016$ & [68] \\
\hline $\begin{array}{l}\text { neoantigenR: An annotation based pipeline } \\
\text { for tumor neoantigen identification from } \\
\text { sequencing data }\end{array}$ & GSS + FASTA & $\begin{array}{l}\text { R package, uses } M H C \text {, } \\
\text { unpublished }\end{array}$ & & [43] \\
\hline
\end{tabular}


neoantigen calls do not try to compute the probability of trimming from ERAP1 (proteosomal cleavage) and peptide processing from TAP1/TAP2, even if some modeling work has been done in the past [44-47]. A few notable exceptions do exist but they start the analysis from preprocessed FASTA files, such as NetTepi (https://www.ncbi.nlm.nih. gov/pubmed/24863339) and NetCTL (https://www.ncbi. nlm.nih.gov/pubmed/20379710), including also a prediction method for $\mathrm{T}$ cell reactivity. It is reasonable to think that the integration of these additional modules would increase the prediction power in terms of specificity.

The effective prediction of immunogenicity can benefit from some additional modeling on the quality of the neoantigen. In this context, an initial hypothesis was formulated in mice where effective neoantigen vaccines were based on a neoantigen with higher binding affinity than the corresponding WT epitope as a means of predicting NetMHC [25]. This feature may spare neoantigens (somatic mutations) from immunological tolerance, which deletes self-reactive T-cells centrally or in periphery. Several papers have explored the immune responses against neoantigens in patients treated with ICI endowed with defined features that better correlate with clinical outcomes. Common sequence motifs similar/homolog to viral epitopes were identified in neoantigens correlating with good prognosis [48]. In accordance with this hypothesis, two bioinformatic papers proposed a "neoantigen fitness model" in order to rank and select the dominant clone-specific neoantigen $[49,50]$. This fitness model is computed by taking into account two main factors: the probability of MHC presentation and T-cell recognition. The first factor is derived from the neoantigen binding affinity, with a matched wild type smoothing factor, as there is indeed a minimal "distance" required from the wild type counterpart. The second factor is computed from the neoantigen similarity with a database of known epitopes. Striking experimental evidence showed effective immunological response against the predicted neoantigens and their viral homolog but not against the neoantigen corresponding self-peptide [49]. These data strongly suggest that quality of neoantigens may have an impact also on the design of an effective $\mathrm{NCV}$, although it remains to be investigated (Fig. 1).

In conclusion, an effective neoantigen prediction pipeline should include: identification of mutations at DNA level, expression from RNA-seq and binding prediction to the MHC of the carrier's HLA and final modeling of neoantigen quality.

\section{Conclusions}

In the last few years, NCVs have entered the arena of immune therapy consequently raising great expectations due to the initial results in preclinical reports and more recently in clinical studies. It is likely that advances in identifying neoantigens as well as a more in depth understanding of cancer resistance mechanisms [51-57] will extend the range of tumor types that are eligible for NCVs treatment. Based on the preclinical and clinical data, the question put forward is: which is the most suitable population for NCVs in the current context of approved drugs? It is clear that low $\mathrm{TH}(\mathrm{TH}-)$ but preexisting immunity, as indicated by the presence of TILs (TILs+), as well as high mutational load, defines the most responsive population to ICI (Fig. 2 upper right quadrant). In contrast, the NCVs approach may be more effective in treating cancers with variants represented at a low allele frequency that respond less to ICI. Induction of a larger repertoire of cancer-specific T-cells by adopting the NCVs approach may lead to a better coverage of $\mathrm{TH}$. The combined action of induced cancer-specific CD8 and CD4 T-cells in the periphery by NCVs is likely to result in higher frequency of TILs in patients cotreated with ICI moving a "cold tumor" from the lower right quadrant to the upper right quadrat of "hot tumors" (Fig. 2). In the clinical world, for instance, these features identify a large number of lung cancer patients that do not respond to pembrolizumab in first or second line treatment [58]. Furthermore in the clinical setting, it has been observed that ICI treatment rescues a limited number of neoantigens-specific T-cells that can be expanded in combination with NCVs [37, 38]. However, for both ICI and NCV approaches, a functional HLA presentation machinery is required, as it would be meaningless to treat a patient with ICI if the $\beta 2$ microglobulin gene is mutated [59]. Induction of an effective T-cell response may be insufficient due to tumor evasion strategies other than PD1 or CTLA-4. A more in-depth knowledge of the tumor microenvironment is therefore

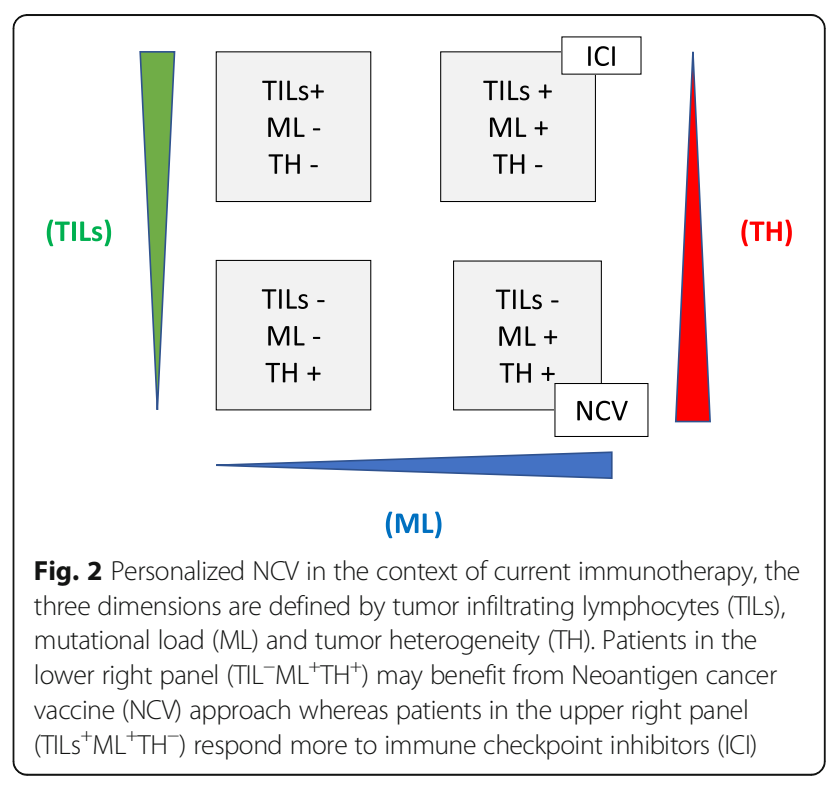


required to deliver the right $\mathrm{NCVs}$ treatment to the right patient in the best responsive conditions.

\section{Perspectives}

It is clear that a single therapeutic approach will not win the battle against a complex and evolving system such as Cancer. Intrinsic factors such as BRAF mutations are associated with a reduced frequency of TILs, which increase upon a short pharmacological intervention in conjunction with ICI [60]. It is reasonable to expect that similar strategies will be effective with NCV. Therapies against a single target leads in most cases to the selection of genetic variants, which invariably lead to tumor relapses. A similar issue was also observed with ICI using anti-PD1 treatments [61]. In line with this concept tumors relapsing under ICI treatment showed a different mutational landscape with a significant selection of a different spectrum of neoeptitope variants [1]. One possible explanation is the suboptimal response against neoantigens [62]. NCVs promise to be a valuable alternative since they can be tailored to target multiple neoepitopes, thus reducing the risk of immune-evasion due to loss of expression of subsets of neoantigens. Furthermore, with the advancement of NGS technologies and with the increasing sensitivity of liquid biopsies it will be possible in the future to design for the same patient sequential NCVs targeting new neoepitopes selected during tumor evolution.

NCVs represent a new form of precision medicine. Several aspects of the NCV approach require further optimization such as the prediction method for CD8 and CD4 neoantigens or the need of new models for clinical trials. Although technically complex and expensive, it offers important advantages. As stated before it is expected to widen the spectrum of patients responsive to ICI and to synergize with it, for example in cases of relapse to ICI treatment, as reported for the three melanoma patients treated with NCVs followed by ICI [37, 38]. In addition, it may offer a practical advantage to currently untreatable patients approach. For instance, a lung cancer patient with no ALK or ROS translocations and EGFR mutations and with a relatively low expression of PD-L1 and a medium to high neoantigen load would be eligible for the adjuvant NCV approach.

Finally, NCVs pose significant manufacturing, regulatory and marketing issues. The authorization process for a new drug is usually based on expensive large scale randomized clinical trials. This is not feasible with individualized therapies such as NCVs. Pleasingly, this paradigm is changing also thanks to the success of CAR-T therapies where, for example in the case of Tisagenlecelucel, FDA approval was obtained based on the (striking) results of a registration trial involving only 63 patients. Individualized therapies such as CAR-T have also set the ground for very high costs. Are NCVs expected follow the same paradigm? And if so, how sustainable are the increasing costs of personalized therapies in financially "stressed" health systems? These are all important questions that need to be addressed to allow our patients access to innovation.

\begin{abstract}
Abbreviations
APC: Antigen presenting cells; CTAG1A also known as NY-ESO-1: Cancertestis antigen; CTAs: Cancer testis antigens; CTLA-4: Cytotoxic T lymphocyte antigen 4; DC: Dendritic cells; FPKM: Fragments Per Kilobase of transcript per Million mapped reads; HBV: Hepatitis B virus; HER2: Epidermal growth factor receptor 2; HLA: Human leukocyte antigen; HPV: Human papillomavirus; ICl: Immune check point inhibitors; MAGE: Melanoma-associated antigen; MART1: Melanoma antigen recognized by T cells; MCC: Merkel cell carcinoma; MHC: Major histocompatibility complex; NCV: Neoantigen cancer vaccine; NGS: Next generation sequencing; NSCLC: Non-small-cell lung cancer; PAP-GMCSF: Granulocyte-macrophage colony-stimulating factor; PD1: Programmed cell death protein 1; PSA: Prostate-specific antigen; RPM: Reads per Million mapped reads; SAGE1: Sarcoma antigen 1; TAAs: Tumor associated antigens; TCR: T cell receptor; TERT: Human telomerase reverse transcriptase; TH: Tumor heterogeneity; TILs: Tumor infiltrating lymphocytes; TSAs: Tumor specific antigens; VCF: Variant calling format
\end{abstract}

\section{Acknowledgements}

L.A. and G.C. work was supported by grants AIRC IG 17827 and IG 15216, respectively. We thank Tania Merlino for editorial assistance.

\section{Funding}

L.A. and G.C. work was supported by grants AIRC IG 17827 and IG 15216, respectively.

\section{Authors' contributions}

F.P. and L.A. conceived and drafted the manuscript and designed the figures, M.P. wrote the bioinformatics paragraph, all the authors contributed in writing and G.C. supervised the manuscript. All authors read and approved the final manuscript.

\section{Ethics approval and consent to participate}

Not applicable

\section{Competing interests}

The authors declare that they have no competing interests.

\section{Publisher's Note}

Springer Nature remains neutral with regard to jurisdictional claims in published maps and institutional affiliations.

\section{Author details}

${ }^{1}$ Takis, Rome, Italy. ${ }^{2}$ Biogem, Ariano Irpino, Italy. ${ }^{3}$ UOSD SAFU, IRCSS Regina Elena National Cancer Institute, Rome, Italy. ${ }^{4}$ Scientific Directorate, IRCCS Regina Elena National Cancer Institute, Rome, Italy. ${ }^{5}$ Alleanza contro il Cancro, Rome, Italy.

Received: 14 March 2018 Accepted: 3 April 2018

Published online: 20 April 2018

\section{References}

1. Anagnostou V, Smith KN, Forde PM, Niknafs N, Bhattacharya R, White J, et al. Evolution of neoantigen landscape during immune checkpoint blockade in non-small cell lung cancer. Cancer Discov. 2017;7:264-76.

2. Alexandrov LB, Nik-Zainal S, Wedge DC, Aparicio SA, Behjati S, Biankin AV, Bignell GR, Bolli N, Borg A, Børresen-Dale AL, Boyault S, Burkhardt B, Butler AP, Caldas C, Davies HR, Desmedt C, Eils R, Eyfjörd JE, Foekens JA, Greaves M, Hosoda F, Hutter B, llicic T, Imbeaud S, Imielinski M, Jäger N, Jones DT, Jones D, Knappskog S, Kool M, Lakhani SR, López-Otín C, Martin S, Munshi NC, Nakamura H, Northcott PA, Pajic M, Papaemmanuil E, Paradiso A, Pearson JV, Puente XS, Raine K, Ramakrishna M, Richardson AL, Richter J, Rosenstiel P, Schlesner M, Schumacher TN, Span PN, Teague JW, Totoki Y, 
Tutt AN, Valdés-Mas R, van Buuren MM, van 't Veer L, Vincent-Salomon A, Waddell N, Yates LR; Australian Pancreatic Cancer Genome Initiative; ICGC Breast Cancer Consortium; ICGC MMML-Seq Consortium; ICGC PedBrain, Zucman-Rossi J, Futreal PA, McDermott U, Lichter P, Meyerson M, Grimmond SM, Siebert R, Campo E, Shibata T, Pfister SM, Campbell PJ, Stratton MR. Signatures of mutational processes in human cancer. Nature. 2013;500(7463):415-21. https://doi.org/10.1038/nature12477. Epub 2013 Aug 14. Erratum in: Nature. 2013 Oct 10;502(7470):258. Imielinsk, Marcin [corrected to Imielinski, Marcin].

3. Zhao B, Sedlak JC, Srinivas R, Creixell P, Pritchard JR, Tidor B, et al. Exploiting temporal collateral sensitivity in tumor clonal evolution. Cell Elsevier Inc. 2016;165:234-46. Available from: http://www.cell.com/cell/fulltext/S00928674(16)30059-9

4. Seliger B, Kloor M, Ferrone S. HLA class II antigen-processing pathway in tumors: Molecular defects and clinical relevance. Oncoimmunology. 2017; 6(2):e1171447. https://doi.org/10.1080/2162402X.2016.1171447.

5. Hu Z, Ott PA, Wu CJ. Towards personalized, tumour-specific, therapeutic vaccines for cancer. Nat. Publ. Gr. Nature Publishing Group; 2017; Available from: https://doi.org/10.1038/nri.2017.131

6. Yarchoan M, Johnson BA 3rd, Lutz ER, Laheru DA, Jaffee EM. Targeting neoantigens to augment antitumour immunity. Nat Rev Cancer England. 2017;17:209-22.

7. Finn OJ, Rammensee H-G. Is it possible to develop Cancer vaccines to Neoantigens, what are the major challenges, and how can these be overcome? United States: Neoantigens: Nothing New in Spite of the Name. Cold Spring Harb. Perspect. Biol; 2017.

8. Brown SD, Warren RL, Gibb EA, Martin SD, Spinelli JJ, Nelson BH, et al. Neoantigens predicted by tumor genome meta-analysis correlate with increased patient survival. Genome Res. 2014;24:743-50.

9. Giannakis M, Mu XJ, Shukla SA, Qian ZR, Cohen O, Nishihara R, et al. Genomic correlates of immune-cell infiltrates in colorectal carcinoma. Cell Rep The Authors. 2016;15:857-65. Available from: https://doi.org/10.1016/j. celrep.2016.03.075

10. Howitt BE, Shukla SA, Sholl LM, Ritterhouse LL, Watkins JC, Rodig S, et al. Association of polymerase e-mutated and microsatellite-instable endometrial cancers with neoantigen load, number of tumor-infiltrating lymphocytes, and expression of PD-1 and PD-L1. JAMA Oncol. 2015;1: 1319-23.

11. Tran E, Robbins PF, Rosenberg SA. Final common pathway' of human cancer immunotherapy: targeting random somatic mutations. Nat Immunol. 2017;18:255-62

12. Whiteside TL, Demaria S, Rodriguez-Ruiz ME, Zarour HM, Melero I. Emerging opportunities and challenges in cancer immunotherapy. Clin Cancer Res. 2016;22:1845-55.

13. Van Allen EM, Miao D, Schilling B, Shukla SA, Blank C, Zimmer L, et al. Genomic correlates of response to CTLA-4 blockade in metastatic melanoma. Science (80-. ). 2015;350:207-11. [cited 2018 Jan 30]; Available from: http://www.ncbi.nlm.nih.gov/pubmed/26359337

14. Rizvi NA, Hellmann MD, Snyder A, Kvistborg P, Makarov V, Havel JJ, et al. Mutational landscape determines sensitivity to PD-1 blockade in non-small cell lung cancer. Science (80-. ). 2015;348:124-128. Available from: http:// www.ncbi.nlm.nih.gov/pubmed/25765070\%5Cn, http://www.sciencemag. org/cgi/doi/10.1126/science.aaa1348

15. Le DT, Durham JN, Smith KN, Wang H, Bartlett BR, Aulakh LK, et al. Mismatch-repair deficiency predicts response of solid tumors to PD-1 blockade. Science 2017;6733:1-11. Available from: http://science. sciencemag.org/content/sci/early/2017/06/07/science.aan6733.full. pdf\%5Cn, http://www.ncbi.nlm.nih.gov/pubmed/28596308

16. McGranahan N, Furness AJS, Rosenthal R, Ramskov S, Lyngaa R, Saini SK, et al. Clonal neoantigens elicit T cell immunoreactivity and sensitivity to immune checkpoint blockade. Science (80- .). 2016;351:1463-9.

17. Strønen E, Toebes M, Kelderman S, Van BMM, Yang W, Van RN, et al. Targeting of cancer neoantigens with donor-derived T cell receptor repertoires. Science (80-. ). 2016;2288:1-11.

18. Tran E, Turcotte S, Gros A, Robbins PF, Lu Y, Dudley ME, et al. Cancer immunotherapy based on mutation-specific CD4+ T cells in a patient with epithelial Cancer. Science (80- ). 2014;9:641-5.

19. Robbins PF, Lu Y-C, El-Gamil M, Li YF, Gross C, Gartner J, et al. Mining exomic sequencing data to identify mutated antigens recognized by adoptively transferred tumor-reactive T cells. Nat Med. 2013;19:747-752. Available from: http:/www.nature.com/nm/journal/v19/n6/full/nm.3161.
html\#methods\%5Cn, http://www.pubmedcentral.nih.gov/articlerender. fcgi?artid $=3757932 \&$ tool=pmcentrez\&rendertype $=$ abstract

20. Castle JC, Kreiter S, Diekmann J, Löwer M, Van De Roemer N, De Graaf J, et al. Exploiting the mutanome for tumor vaccination. Cancer Res. 2012;72:1081-91.

21. Liu XS, Mardis ER. Applications of Immunogenomics to Cancer. Cell. 2017; 168:600-12.

22. Kreiter $\mathrm{S}$, Vormehr $\mathrm{M}$, van de Roemer $\mathrm{N}$, Diken $\mathrm{M}$, Löwer $\mathrm{M}$, Diekmann J, et al. Mutant MHC class II epitopes drive therapeutic immune responses to cancer. Nature 2015;520:692-696. Available from: http://www.nature. com/doifinder/10.1038/nature14426\%5Cn, http://dx.doi.org/10.1038/ nature 14426

23. Yadav M, Jhunjhunwala S, Phung QT, Lupardus P, Tanguay J, Bumbaca S, et al. Predicting immunogenic tumour mutations by combining mass spectrometry and exome sequencing. Nature 2014;515:572-576. Available from: http://www.nature.com/doifinder/10.1038/nature $14001 \% 5$ Cn, http:// www.ncbi.nIm.nih.gov/pubmed/25428506

24. Kuai R, Ochyl LJ, Bahjat KS, Schwendeman A, Moon JJ. Designer vaccine nanodiscs for personalized cancer immunotherapy. Nat Mater England. 2017;16:489-96.

25. Duan F, Duitama J, Al Seesi S, Ayres CM, Corcelli S a, Pawashe AP, et al. Genomic and bioinformatic profiling of mutational neoepitopes reveals new rules to predict anticancer immunogenicity. J Exp Med 2014;211:2231-2248. Available from: http://www.pubmedcentral.nih.gov/articlerender.fcgi?artid= 4203949\&tool=pmcentrez\&rendertype=abstract\%5Cn, http://www.ncbi.nlm. nih.gov/pubmed/25245761\%5Cn, http://www.pubmedcentral.nih.gov/ articlerender.fcgi?artid=PMC4203949

26. Gubin MM, Zhang X, Schuster H, Caron E, Ward JP, Noguchi T, et al. Checkpoint blockade cancer immunotherapy targets tumour-specific mutant antigens. Nature [Internet]. 2014;515:577-81. Available from: http:// www.pubmedcentral.nih.gov/articlerender.fcgi?artid=4279952\&tool= pmcentrez\&rendertype $=$ abstract.

27. Schumacher T, Bunse L, Pusch S, Sahm F, Wiestler B, Quandt J, et al. A vaccine targeting mutant IDH1 induces antitumour immunity. Nature 2014; 512:324-327. Available from: http://www.nature.com/doifinder/10.1038/ nature13387\%5Cn, http://www.ncbi.n/m.nih.gov/pubmed/25043048\%5Cn, http://www.nature.com/doifinder/10.1038/nature13387

28. Kranz LM, Diken M, Haas H, Kreiter S, Loquai C, Reuter KC, et al. Systemic RNA delivery to dendritic cells exploits antiviral defence for cancer immunotherapy. Nature. 2016;534:396-401.

29. Kuai R, Ochyl LJ, Bahjat KS, Schwendeman A, Moon JJ. Cancer immunotherapy; 2016. p. 1

30. Martin SD, Brown SD, Wick DA, Nielsen JS, Kroeger DR, Twumasi-Boateng K, et al. Low mutation burden in ovarian cancer may limit the utility of neoantigen-targeted vaccines. PLoS One. 2016;11:1-22. Available from: https://doi.org/10.1371/journal.pone.0155189

31. Zolkind P, Przybylski D, Marjanovic N, Nguyen L, Johanns T, Alexandrov A, et al. Cancer immunogenomic approach to neoantigen discovery in a checkpoint blockade responsive murine model of oral cavity squamous cell carcinoma. Oncotarget. 2018:9(3):4109-4119. https://doi.org/10.18632/ oncotarget.23751

32. Kim TJ, Jin HT, Hur SY, Yang HG, Seo YB, Hong SR, Lee CW, Kim S, Woo JW, Park KS, Hwang YY, Park J, Lee IH, Lim KT, Lee KH, Jeong MS, Surh CD, Suh YS, Park JS, Sung YC. Clearance of persistent HPV infection and cervical lesion by therapeutic DNA vaccine in CIN3 patients. Nat Commun. 2014;5: 5317. https://doi.org/10.1038/ncomms6317. PMID:25354725

33. Trimble CL, Morrow MP, Kraynyak KA, Shen X, Dallas M, Yan J, et al. Safety, efficacy, and immunogenicity of VGX-3100, a therapeutic synthetic DNA vaccine targeting human papillomavirus 16 and 18 E6 and E7 proteins for cervical intraepithelial neoplasia 2/3: a randomised, double-blind, placebocontrolled phase 2b trial. Lancet. 2015;386:2078-88.

34. Morrow MP, Kraynyak KA, Sylvester AJ, Dallas M, Knoblock D, Boyer JD, et al. Clinical and immunologic biomarkers for histologic regression of high-grade cervical dysplasia and clearance of HPV16 and HPV18 after immunotherapy. Clin Cancer Res. 2018;24:276-94.

35. Diaz-Montero CM, Chiappori A, Aurisicchio L, Bagchi A, Clark J, Dubey S, et al. Phase 1 studies of the safety and immunogenicity of electroporated HER2/CEA DNA vaccine followed by adenoviral boost immunization in patients with solid tumors. J Transl Med. 2013;11:1. Available from: Journal of Translational Medicine

36. Carreno BM, Magrini V, Becker-Hapak M, Kaabinejadian S, Hundal J, Petti AA, et al. A dendritic cell vaccine increases the breadth and diversity of 
melanoma neoantigen-specific T cells. Science (80- ) . 2015;348:803-8. Available from: http://www.sciencemag.org/content/348/6236/803.full

37. Ott PA, Hu Z, Keskin DB, Shukla SA, Sun J, Bozym DJ, et al. An immunogenic personal neoantigen vaccine for patients with melanoma. Nature. 2017;547: 217-21. Nature Publishing Group Available from: https://doi.org/10.1038/ nature22991

38. Sahin U, Derhovanessian E, Miller M, Kloke BP, Simon P, Löwer M, et al. Personalized RNA mutanome vaccines mobilize poly-specific therapeutic immunity against cancer. Nature. 2017;547:222-6. Nature Publishing Group Available from: https://doi.org/10.1038/nature23003

39. Castel SE, Levy-Moonshine A, Mohammadi P, Banks E, Lappalainen T. Tools and best practices for data processing in allelic expression analysis. Genome Biol. 2015;16:195. [cited 2018 Feb 11]Available from: http://genomebiology. com/2015/16/1/195

40. Boegel $S$, Löwer $M$, Schäfer $M$, Bukur T, de Graaf J, Boisguérin $V$, et al. HLA typing from RNA-Seq sequence reads. Genome Med. 2012;4:102. [cited 2018 Feb 11]Available from: http://genomemedicine.biomedcentral.com/articles/ 10.1186/gm403

41. Warren RL, Choe G, Freeman DJ, Castellarin M, Munro S, Moore R, et al. Derivation of HLA types from shotgun sequence datasets. Genome Med. 2012;4:95. [cited $2018 \mathrm{Feb}$ 11] Available from: http://genomemedicine. biomedcentral.com/articles/10.1186/gm396

42. Shukla SA, Rooney MS, Rajasagi M, Tiao G, Dixon PM, Lawrence MS, et al. Comprehensive analysis of cancer-associated somatic mutations in class I HLA genes. Nat Biotechnol. 2015;33:1152-8. [cited 2018 Feb 11] Available from: http://www.nature.com/articles/nbt.3344

43. Tang S, Madhavan S. neoantigenR: an annotation based pipeline for tumor neoantigen identification from sequencing data. bioRxiv. 2017; Available from: http://biorxiv.org/content/early/2017/08/08/171843.abstract

44. Bhasin M, Raghava GPS. Analysis and prediction of affinity of TAP binding peptides using cascade SVM. Protein Sci. 2004;13:596-607. Cold Spring Harbor Laboratory Press Available from: http://www.ncbi.nlm.nih.gov/pmc/ articles/PMC2286721/

45. Hearn A, York IA, Rock KL. The specificity of trimming of MHC class I-presented peptides in the endoplasmic reticulum. J Immunol. 2009;183:5526-36. Available from: http://www.ncbi.nlm.nih.gov/pmc/articles/PMC2855122/

46. Zhang GL, Petrovsky N, Kwoh CK, August JT, Brusic V. PRED(TAP): a system for prediction of peptide binding to the human transporter associated with antigen processing. Immunome res. London: BioMed central. 2006;2:3. Available from: http://www.ncbi.nlm.nih.gov/pmc/articles/PMC1524936/

47. Chen H, Li L, Weimershaus M, Evnouchidou I, van Endert P, Bouvier M. ERAP1-ERAP2 dimers trim MHC I-bound precursor peptides; implications for understanding peptide editing. Sci Rep. 2016;6:28902. Nature Publishing Group. Available from: http://www.ncbi.nlm.nih.gov/pmc/articles/ PMC4981824/

48. Snyder A, Makarov V, Merghoub T, Yuan J, Zaretsky JM, Desrichard A, et al. Genetic basis for clinical response to CTLA-4 blockade in melanoma. N Engl J Med. 2014;2189-2199. Available from: http://www.ncbi.nlm.nih.gov/ pubmed/25409260.

49. Balachandran VP, Luksza M, Zhao JN, Makarov V, Moral JA, Remark R, et al. Identification of unique neoantigen qualities in long-term survivors of pancreatic cancer. Nature. England. 2017;551:512-6.

50. Luksza M, Riaz N, Makarov V, Balachandran VP, Hellmann MD, Solovyov A, et al. A neoantigen fitness model predicts tumour response to checkpoint blockade immunotherapy. Nature England. 2017;551:517-20.

51. Peng W, Chen JQ, Liu C, Malu S, Creasy C, Tetzlaff MT, et al. Loss of PTEN promotes resistance to T cell-mediated immunotherapy. Cancer Discov. 2016;6:202-16.

52. Rizvi NA, Chan TA. Immunotherapy and oncogenic pathways: the PTEN connection. Cancer Discov. 2016;6:128-9.

53. Milella M, Falcone I, Conciatori F, Matteoni S, Sacconi A, De Luca T, et al. PTEN status is a crucial determinant of the functional outcome of combined MEK and MTOR inhibition in cancer. Sci Rep. 2017;7:1-15.

54. Hugo W, Zaretsky JM, Sun L, Song C, Moreno BH, Hu-Lieskovan S, et al. Genomic and transcriptomic features of response to anti-PD-1 therapy in metastatic melanoma. Cell Elsevier Inc. 2016;165:35-44.

55. George S, Miao D, Demetri GD, Adeegbe D, Rodig SJ, Shukla S, et al. Loss of PTEN is associated with resistance to anti-PD-1 checkpoint blockade therapy in metastatic uterine Leiomyosarcoma. Immunity Elsevier Inc. 2017; 46:197-204.
56. Gao J, Shi LZ, Zhao H, Chen J, Xiong L, He Q, et al. Loss of IFN-gamma pathway genes in tumor cells as a mechanism of resistance to anti-CTLA-4 therapy. Cell. 2016;167:397-404.e9.

57. Charoentong P, Finotello F, Angelova M, Mayer C, Efremova M, Rieder D, et al. Pan-cancer Immunogenomic analyses reveal genotypeImmunophenotype relationships and predictors of response to checkpoint blockade. Cell Rep ElsevierCompany. 2017;18:248-62.

58. Peters S, Kerr KM, Stahel R. PD-1 blockade in advanced NSCLC: a focus on pembrolizumab. Cancer Treat Rev. 2018;62:39-49.

59. Sade-Feldman M, Jiao YJ, Chen JH, Rooney MS, Barzily-Rokni M, Eliane JP, et al. Resistance to checkpoint blockade therapy through inactivation of antigen presentation. Nat. Commun. 2017;8

60. Deken MA, Gadiot J, Jordanova ES, Lacroix R, van Gool M, Kroon P, et al. Targeting the MAPK and PI3K pathways in combination with PD1 blockade in melanoma. Oncoimmunology. United States. 2016:5:e1238557.

61. Mcgranahan N, Furness AJS, Rosenthal R, Ramskov S, Lyngaa R, Saini SK, et al. Clonal neoantigens elicit T cell immunoreactivity and sensitivity to immune checkpoint blockade. Science (80-. ). 2016;351:1463-9. Available from: http://www.sciencemag.org/cgi/doi/10.1126/science.aaf1490

62. Strønen E, Toebes M, Kelderman S, Buuren MM Van, Yang W, Rooij N Van, et al. Targeting of cancer neoantigens with donor-derived T cell receptor repertoires(1). 2016;2288:1-11.

63. Gubin MM, Zhang X, Schuster H, Caron E, Ward JP, Noguchi T, et al. Checkpoint blockade cancer immunotherapy targets tumour-specific mutant antigens. Nature. 2014;515:577-81. Available from: http://www. pubmedcentral.nih.gov/articlerender.fcgi?artid=4279952\&tool= pmcentrez\&rendertype $=$ abstract

64. Tappeiner E, Finotello F, Charoentong P, Mayer C, Rieder D, Trajanoski Z. TIminer: NGS data mining pipeline for cancer immunology and immunotherapy. Bioinformatics. 2017;33:3140-1. [cited 2018 Feb 11] Available from: http://academic.oup.com/bioinformatics/article/33/19/3140/ 3868722

65. Bais P, Namburi S, Gatti DM, Zhang X, Chuang JH. CloudNeo: a cloud pipeline for identifying patient-specific tumor neoantigens. Bioinformatics. 2017;33:3110-2. [cited 2018 Feb 11] Available from: http://academic.oup. com/bioinformatics/article/33/19/3110/3866879/CloudNeo-a-cloud-pipelinefor-identifying

66. Zhou Z, Lyu X, Wu J, Yang X, Wu S, Zhou J, et al. TSNAD: an integrated software for cancer somatic mutation and tumour-specific neoantigen detection. R Soc open Sci. 2017:4:170050. [cited 2018 Feb 11] Available from: http://rsos.royalsocietypublishing.org/lookup/doi/10.1098/rsos.170050

67. Zhang J, Mardis ER, Maher CA. INTEGRATE-neo: a pipeline for personalized gene fusion neoantigen discovery. Bioinformatics England. 2017;33:555-7.

68. Hundal J, Carreno BM, Petti AA, Linette GP, Griffith OL, Mardis ER, et al. pVAC-Seq: a genome-guided in silico approach to identifying tumor neoantigens. Genome Med. 2016;8:11. [cited 2018 Feb 11] Available from: http://genomemedicine.com/content/8/1/11

\section{Ready to submit your research? Choose BMC and benefit from:}

- fast, convenient online submission

- thorough peer review by experienced researchers in your field

- rapid publication on acceptance

- support for research data, including large and complex data types

- gold Open Access which fosters wider collaboration and increased citations

- maximum visibility for your research: over $100 \mathrm{M}$ website views per year

At BMC, research is always in progress.

Learn more biomedcentral.com/submissions 